\title{
Surface roughness effect on thermohydrodynamic analysis of journal bearings lubricated with couple stress fluids
}

https://doi.org/10.1515/nleng-2018-0017

Received October 7, 2017; revised May 18, 2018; accepted July 6, 2018.

\begin{abstract}
The paper presents, surface roughness effect for thermo-hydrodynamic analysis of journal bearings extended to couple stress lubricants with high polymer additives. A modified energy equation is simultaneously solved with heat transfer equation as well as modified Reynolds equation by using Multigrid method. The effects of couple stress and surface roughness on the performances of a finite journal bearing are presented in detail. Further, it is shown that lubricants with couple stress and surface roughness, not only increases the load capacity and decreases the friction coefficient, but also generates a lower bearing temperature field. Thus, the lubricant with couple stress improves the performance of journal bearings. The characteristics of bearing are compared with numerical results.
\end{abstract}

\section{Introduction}

The performance of journal bearings with thermal effects in lubrication process is analysed by thermal hydrodynamic lubrication (THD). Since it produces peak bearing temperature, bearing failure can be estimated at the design when maximum temperature exceeds a certain limit. Ferron et al. [1] solved Dowson's [2] generalized Reynolds equation simultaneously with energy equation and heat transfer equation and produced excellent results. THD solutions under various boundary conditions, considering the mixing of recirculation of fluid and supply oil is obtained by Khonsari and Beaman [3]. Ott and Paradissiadis [4] applied Jakobsson-Floberg-Olsson (JFO)

\footnotetext{
*Corresponding Author: Vishwanath B. Awati, Rani Channamma University, NH-4 Bhootaramanahatti Belagavi, 591156, India, E-mail: awati_vb@yahoo.com

Ashwini Kengangutti, Belagavi, 591156, India, E-mail:

ashwini7.k9@gmail.com
}

boundary conditions, which conserve mass within cavitated region as well as boundary, to determine the cavitation region and used same energy equation for both full film and cavitation regions. In recent years, more comprehensive THD analysis are developed by Paranjpe and Han [5], Khonsari et al. [6], Ramesh et al. [7], and Shi and Wang [8]. However all these studies do not include more complex fluids. The use of complex fluids as lubricants is getting more and more important to fulfil the desires of modern machines. For example, high polymer additives are added in lubricating oils as a kind of viscosity index improver so that viscosity variation with temperature decreases. On the other hand, addition of polymers exhibit non-newtonian behaviour, leading to classical continuum theory which becomes inapplicable.

The classical continuum fluid mechanics neglects size of fluid particles to describe the flow of fluids. The microcontinuum fluid mechanics accounts for intrinsic motion of material constituents in order to extend the range of application, for example, to describe the flow of complex fluids, polymer molecules are used in polymeric suspensions. The classical continuum fluid mechanics is generalized by non-polar theory of fluids, which is characterized by classical Cauchy stress. The micro-continuum fluid mechanics is generalized by polar theory of complex fluids, which is characterized by classical Cauchy stress as well as by couple stress [9]. Recently several studies are carried out to describe the flow of complex fluids. The couple stress theory of fluids was presented by Stokes [10] to examine simplest generalization of classical theory that would allow for polar effects. This model defines the rotation field in terms of velocity field; the rotation vector is equated to one-half of curl of velocity vector. The couple stress fluid model has been widely used in lubrication for its relative mathematical simplicity. A study of a journal bearing lubricated with a couple stress fluids including elasticity of the linear is presented by Mokhiamer et al. [11]. This showed that the influence of couple stress fluid on the bearing characteristics is significantly apparent and that lubricants with couple stresses produce an increase in the load carrying capacity, decrease in attitude angle, the frictional force and 
side leakage. Lin [12] analysed the study of squeeze film characteristics of finite journal bearings assuming the lubricant to be an incompressible fluid with couple stress. The studies showed that couple stress effects provide a longer time to prevent journal bearing contact and resulting in longer bearing life. Das [13] studied the slider bearings lubricated with couple stress fluids in a magnetic field and observed that maximum load capacity increases with an increase in both magnetic and couple stress parameters. Also, the ratio of inlet to outlet film heights for maximum load capacity increases with magnetic parameter and decreases with couple stress parameter.

In most of the theoretical studies of film lubrication it was more or less assumed that the bearing surfaces could be presented by smooth mathematical planes. But this appears to be an unrealistic assumption particularly in bearing, working with small film thickness. However, bearing surfaces particularly after they have received some run in and wear, seldom exhibit a type of roughness approximated by this model. Christensen and Tonder investigated the hydrodynamic lubrication of rough surfaces having finite width. In 1975 Christensen et al. [17] obtained a generalised Reynolds equation applicable to rough surface by assuming that the film thickness function is governing a stochastic process. Bujurke et al. [14] studied the effect of surface roughness on squeeze film poroelastic bearings with special reference to synovial joints. A simplified mathematical model is developed for understanding combined effect of surface roughness and couple stress. Surface roughness effects in a short porous journal bearing with a couple stress fluid is studied by Naduvinamani et al. [15]. Thermohydrodynamic analysis of journal bearings with couple stress fluids is analysed by Xiao et al. [16]. All the applications of couple stress models are limited to isothermal case as noticed up to now.

Multigrid method is used to find the solution more accurately than the finite difference method which used in earlier study. Hydrodynamic bearings are usually assumed to have a smooth surface. For most practical bearings this is a good approximation since the influence of the roughness on the bearing performance is negligible. Under normal operating conditions the surface roughness heights are small compared to the thickness of the lubrication film. However, when surface roughness amplitudes are of the same range as that of lubrication film thickness, the surface roughness effects can no longer be neglected. Since most of the available literature on micro asperity lubrication is based on the smooth surface assumption, the present work extends the existing literature to cover the effect of random surface roughness on micro asperity hydrodynamic lubrication. The aim of this paper is to present the
THD performance of journal bearings lubricated with couple stress fluids with surface roughness effect. The energy equation together with heat transfer equation and modified Reynolds equation were numerically solved by using multigrid method. The various effects which include temperature field, load carrying capacity, friction force, friction coefficient and end leakage are analysed in detail for different couple stress parameters.

\section{Theoretical analysis}

On the basis of Micro-continuum theory of Stokes [10], body forces and body couples are neglected; the basic equations of motion of fluids with couple stresses can be expressed as

$$
\begin{gathered}
\dot{\rho}+\rho v_{i, i}=0 \\
\rho \dot{v}_{i}=\sigma_{j i, j} \\
M_{j i, j}+e_{i j k} \sigma_{j k}=0
\end{gathered}
$$

The constitutive equations for stress tensor and couple stress tensor are given as [10]

$$
\begin{gathered}
\sigma_{i j}=\left(-p+\lambda v_{k, k}\right) \delta_{i j}+\mu\left(v_{i, j}+v_{j, i}\right)-\frac{1}{2} e_{i j k} M_{r k, r} \\
M_{r k}=\frac{1}{3} M_{n n} \delta_{r k}+4 \eta \omega_{k, r}+4 \eta^{\prime} \omega_{r, k}
\end{gathered}
$$

where $\lambda=-2 \mu / 3$. A finite journal bearing lubricated with an incompressible couple stress fluid is considered and is shown in the Fig. 1. The mathematical formulation for film thickness with surface roughness is

$$
H=h+h_{s}=C(1+\varepsilon \cos \theta)+h_{s}(\theta, z, \xi)
$$

By the assumptions of hydrodynamic lubrication to thin films, the equations of motion are reduced as

$$
\begin{gathered}
\frac{\partial p}{\partial x}=\mu \frac{\partial^{2} u}{\partial y^{2}}-\eta \frac{\partial^{4} u}{\partial y^{4}} \\
\frac{\partial p}{\partial y}=0 \\
\frac{\partial p}{\partial z}=\mu \frac{\partial^{2} w}{\partial y^{2}}-\eta \frac{\partial^{4} w}{\partial y^{4}}
\end{gathered}
$$

The velocity boundary conditions at the shaft surface are

$$
\begin{aligned}
& \left.u\right|_{y=0}=U,\left.v\right|_{y=0}=0,\left.w\right|_{y=0}=0, \\
& \left.\frac{\partial^{2} u}{\partial y^{2}}\right|_{y=0}=0,\left.\frac{\partial^{2} w}{\partial y^{2}}\right|_{y=0}=0
\end{aligned}
$$




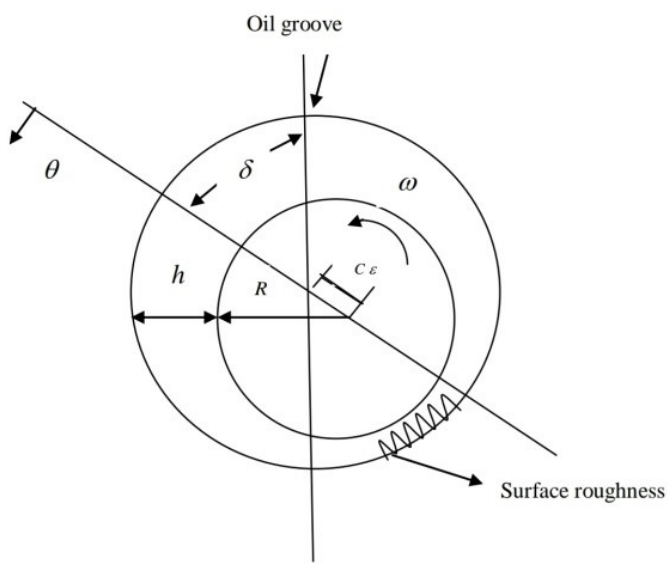

Fig. 1: The schematic diagram of journal bearing

The velocity boundary conditions at the bushing surface are

$$
\begin{aligned}
& \left.u\right|_{y=H}=0,\left.v\right|_{y=H}=0,\left.w\right|_{y=H}=0, \\
& \left.\frac{\partial^{2} u}{\partial y^{2}}\right|_{y=H}=0,\left.\frac{\partial^{2} w}{\partial y^{2}}\right|_{y=H}=0
\end{aligned}
$$

Integrating Eqs. (7a) and (7c) with above boundary conditions, the velocity components can be obtained as

$$
\begin{aligned}
& u=U\left(1-\frac{y}{H}\right)+\frac{1}{2 \mu} \frac{\partial p}{\partial x}\{y(y-H) \\
& \left.+2 l^{2}\left[1-\left(\cosh \frac{2 y-H}{2 l}\right)\left(\cosh \frac{H}{2 l}\right)^{-1}\right]\right\} \\
& w=\frac{1}{2 \mu} \frac{\partial p}{\partial z}\{y(y-H) \\
& \left.+2 l^{2}\left[1-\left(\cosh \frac{2 y-H}{2 l}\right)\left(\cosh \frac{H}{2 l}\right)^{-1}\right]\right\}
\end{aligned}
$$

where $l=\sqrt{\eta / \mu}$

Substituting Eqs. (7) and (8) into Eq. (1), we get the following modified Reynolds equation in the form

$$
\frac{\partial}{\partial x}\left[\frac{f(l, H)}{\mu} \frac{\partial p}{\partial x}\right]+\frac{\partial}{\partial z}\left[\frac{f(l, H)}{\mu} \frac{\partial p}{\partial z}\right]=6 U \frac{\partial H}{\partial x}
$$

where

$$
f(l, H)=H^{3}-12 l^{2} H+24 l^{3} \tanh \left(\frac{H}{2 l}\right) .
$$

Let $f\left(h_{s}\right)$ be the probability density function of stochastic film thickness $h_{s}$. Taking stochastic average of Eq. (10) with respect to $f\left(h_{s}\right)$, we get

$$
\begin{aligned}
& \frac{\partial}{\partial x}\left[E\left(\frac{f(l, H)}{\mu}\right) \frac{\partial E(p)}{\partial x}\right]+\frac{\partial}{\partial z}\left[E\left(\frac{f(l, H)}{\mu}\right) \frac{\partial E(p)}{\partial z}\right] \\
& =6 U E\left(\frac{\partial H}{\partial x}\right)
\end{aligned}
$$

where expectancy operator $\mathrm{E}(\bullet)$ is defined by

$$
E(\bullet)=\int_{-\infty}^{\infty}(\bullet) f\left(h_{s}\right) d h_{s} .
$$

The probability density function [17] is given as

$$
f\left(h_{s}\right)= \begin{cases}\frac{35}{32 c^{7}}\left(c^{2}-h_{s}^{2}\right)^{3}, & -c<h_{s}<c \\ 0, & \text { elsewhere }\end{cases}
$$

where $c=3 \sigma_{1}$.

This non dimensional modified Reynolds equation based on a couple stress model [20] can be obtained by introducing following dimensionless variables

$$
\begin{gathered}
\theta=\frac{x}{R}, y^{\star}=\frac{y}{H}, z^{\star}=\frac{z}{R}, E\left(H^{\star}\right)=\frac{E(H)}{C}=1+\varepsilon \cos \theta, \\
\mu=\frac{\mu}{\mu_{0}}, \omega=\frac{U}{R}, E\left(p^{\star}\right)=\frac{E(p) C^{2}}{\mu_{0} \omega R^{2}}, l^{\star}=\frac{l}{C} .
\end{gathered}
$$

The dimensionless form of modified Reynolds equation can be written as

$$
\begin{aligned}
& \frac{\partial}{\partial \theta}\left[E\left(\frac{f^{\star}\left(l^{\star}, H^{\star}\right)}{\mu_{a v}^{\star}}\right) \frac{\partial E\left(p^{\star}\right)}{\partial \theta}\right]+\frac{\partial}{\partial z^{\star}}\left[E\left(\frac{f^{\star}\left(l^{\star}, H^{\star}\right)}{\mu_{a v}^{\star}}\right) \frac{\partial E\left(p^{\star}\right)}{\partial z^{\star}}\right] \\
& =-6 E(\varepsilon \sin \theta)
\end{aligned}
$$

where

$$
\begin{aligned}
& E\left(f^{\star}\left(l^{\star}, H^{\star}\right)\right)=E\left(H^{\star 3}\right)-12 l^{2} E\left(H^{\star}\right) \\
& +24 l^{\star 3} \tanh \left\{\left(\frac{E\left(H^{\star}\right)}{2 l^{\star}}\right)\right\} .
\end{aligned}
$$

The lubricant viscosity $\mu_{a v}^{\star}$ in Eq. (13) is a cross-film average value. Lubrication in a steady-state and an incompressible couple stress fluid is considered as shown in Fig. 1. According to Tucker and Keogh [18], the viscosity cross film average can offer a close approximation for viscosity in lubrication analysis.

The pressure boundary conditions are:

$$
\begin{aligned}
& \left.\frac{\partial E\left(p^{\star}\right)}{\partial z^{\star}}\right|_{z^{\star}=0}=\left.E\left(p^{\star}\right)\right|_{z^{*}= \pm B / 2 R}=0,\left.E\left(p^{\star}\right)\right|_{\theta=-\delta}=E\left(p_{0}^{*}\right) \\
& \left.\frac{\partial E\left(p^{\star}\right)}{\partial \theta}\right|_{\theta=\theta_{c a v}}=\left.E\left(p^{*}\right)\right|_{\theta=\theta_{c a v}}=0
\end{aligned}
$$

where $\theta_{\text {cav }}$ represents location of starting point for the cavitation zone.

The first law of thermodynamics gives energy equation in fluids and is written as

$$
\rho \frac{D E}{D t}=\nabla \cdot(k \nabla T)+\Phi-p \nabla \cdot \vec{V}
$$

where $E$ is the specific internal energy of fluid and $\Phi$ is the heat dissipation, it can be written as

$$
\Phi=p \nabla \cdot \vec{V}+\overrightarrow{\vec{\sigma}}: \nabla \vec{V} .
$$


Substituting Eqs. (4) and (5) in Eq. (17), we get

$$
\begin{gathered}
\Phi=\lambda\left(\nabla_{i} V_{i}\right)^{2}+\frac{1}{2} \mu\left(\nabla_{i} V_{j}+\nabla_{j} V_{i}\right)^{2} \\
-\eta\left(\nabla_{r} \nabla_{r} e_{k m n} \nabla_{m} V_{n}\right)\left(e_{k i j} \nabla_{i} V_{j}\right)
\end{gathered}
$$

Considering an incompressible couple stress fluid and lubrication in steady state, Eqs. (16) and (18) can be written as

$$
\rho c_{f}\left(u \frac{\partial T}{\partial x}+v \frac{\partial T}{\partial y}+w \frac{\partial T}{\partial z}\right)=K_{f} \frac{\partial^{2} T}{\partial y^{2}}+\Phi
$$

where

$$
\Phi=\mu\left\{\left[\left(\frac{\partial u}{\partial y}\right)^{2}+\left(\frac{\partial w}{\partial y}\right)^{2}\right]-l^{2}\left(\frac{\partial u}{\partial y} \frac{\partial^{3} u}{\partial y^{3}}+\frac{\partial w}{\partial y} \frac{\partial^{3} w}{\partial w^{2}}\right)\right\}
$$

when $l$ tends to zero, Eq. (20) becomes

$$
\Phi=\mu\left[\left(\frac{\partial u}{\partial y}\right)^{2}+\left(\frac{\partial w}{\partial y}\right)^{2}\right]
$$

The non dimensional modified energy equation can be obtained by introducing following dimensionless variables as

$$
u^{\star}=\frac{u}{U}, w^{\star}=\frac{w}{U}, T^{\star}=\frac{T}{T_{0}}, \rho^{\star}=\frac{\rho}{\rho_{0}} .
$$

and the derivatives of $u^{\star}, w^{\star}$ are given in Appendix A.

The dimensionless form of energy equation becomes

$$
\begin{aligned}
& P_{e}\left(\rho^{\star} u^{\star} \frac{\partial E\left(T^{\star}\right)}{\partial \theta}+\Gamma^{\star} \frac{\partial E\left(T^{\star}\right)}{\partial y^{\star}}+\rho^{\star} w^{\star} \frac{\partial E\left(T^{\star}\right)}{\partial z^{\star}}\right) \\
& =\frac{1}{E\left(H^{\star 2}\right)} \frac{\partial^{2} E\left(T^{\star}\right)}{\partial y^{\star 2}}+\Phi^{\star}
\end{aligned}
$$

where $P_{e}=\frac{\rho_{0} c_{f} U C^{2}}{K_{f} R}$

$E\left(\Gamma^{\star}\right)=$

$-\frac{1}{E\left(H^{\star}\right)}\left[\frac{\partial}{\partial x^{\star}}\left(E\left(H^{\star}\right) \int_{0}^{y^{\star}} \rho^{\star} u^{\star} d y^{\star}\right)+\frac{\partial}{\partial z^{\star}}\left(E\left(H^{\star}\right) \int_{0}^{y^{\star}} \rho^{\star} w^{\star} d y^{\star}\right)\right]$

$$
\beta=\frac{\mu_{0} U^{2}}{K_{f} T_{0}}
$$

\section{Discretization of modified Reynolds and Energy equations}

The modified Reynolds equation and energy equation are solved numerically by using one of the most efficient finite difference method i.e. multigrid method. By using second order finite difference scheme, the derivative terms in
Eqs. (13) and (19) can be approximated as

$$
P_{i, j}=A_{1} P_{i+1, j}+A_{2} P_{i-1, j}+A_{3} P_{i, j+1}+A_{4} P_{i, j-1}+A_{5}
$$

where

$$
\begin{gathered}
A_{0}=r^{2}\left(f_{i+0.5, j}+f_{i=0.5, j}+f_{i, j+0.5}+f_{i, j-0.5}\right) \\
A_{1}=f_{i+0.5, j} / \mu_{i+0.5, j} A_{0}, A_{2}=f_{i-0.5, j} / \mu_{i-0.5, j} A_{0}, \\
A_{3}=f_{i, j+0.5} / \mu_{i, j-0.5} A_{0}, A_{4}=f_{i, j=0.5} / \mu_{i, j=0.5} A_{0}
\end{gathered}
$$

$$
A_{5}=-6 \Delta z^{\star 2} \varepsilon_{i} \cos \theta_{i}, r=\frac{\Delta z^{\star}}{\Delta \theta}
$$

The discretized boundary conditions becomes

$$
p_{i, j}-p_{i-1, j}=0, p_{i, j}=0, p_{i, j}=p_{0_{i, j}}
$$

The energy equation can be written in discretized form as

$$
P_{e}\left(\rho^{\star} \mu^{\star} \frac{T_{i, j}^{\star}-T_{i-1, j}^{\star}}{\theta_{i}-\theta_{i-1}}+\Gamma^{\star} \frac{T_{i, j}^{\star}-T_{i-1, j}^{\star}}{y_{i}-y_{i-1}}+\rho^{\star} w^{\star} \frac{T_{i, j}^{\star}-T_{i-1, j}^{\star}}{z_{i}-z_{i-1}}\right)
$$$$
=\frac{1}{H_{i, j}^{\star 2}-H_{i-1, j}^{\star 2}} \frac{T_{i, j}^{\star 2}-T_{i-1, j}^{\star 2}}{y_{i}^{2}-y_{i-1}^{2}}+\Phi_{i}^{\star}
$$

The discretized temperature boundary conditions are

$$
T_{i, j}=T_{\text {mix }_{i, j}}, T_{i, j}-T_{i, j-1}=0
$$

\subsection{Heat transfer equation}

The heat transfer equation in bush for a steadily loaded journal bearing is defined as

$$
\frac{\partial^{2} E\left(T^{\star}\right)}{\partial r^{\star 2}}+\frac{1}{r^{\star}} \frac{\partial E\left(T^{\star}\right)}{\partial r^{\star}}+\frac{1}{r^{\star 2}} \frac{\partial E\left(T^{\star}\right)}{\partial \theta^{2}}+\frac{\partial^{2} E\left(T^{\star}\right)}{\partial z^{\star 2}}=0
$$

The temperature boundary conditions at inlet groove and central plane are respectively given as

$$
E(T)^{\star}\left\|_{\theta=-\delta}=E\left(T_{\text {mix }}^{\star}\right), \frac{\partial E\left(T^{\star}\right)}{\partial z^{\star}}\right\|_{z^{\star}=0}=0
$$

where $E\left(T_{m i x}^{\star}\right)$ is defined as homogeneous mixing temperature of re-circulating oil [3].

At the oil-shaft interface and oil-bush interface, the boundary conditions for THD becomes

$$
\begin{gathered}
q^{\star}=\int_{0}^{2 \pi} \frac{1}{H^{\star}} \frac{\partial T^{\star}}{\partial y^{\star}} \|_{y^{\star}=y} d \theta=0 \\
\frac{\partial T^{\star}}{\partial r^{\star}}\left\|_{r^{\star}=1}=\frac{K(\theta) R}{K_{b} H^{\star}} \frac{\partial T^{\star}}{\partial y^{\star}}\right\|_{y^{\star}=1}
\end{gathered}
$$


where $K(\theta)$ is the thermal conductivity of fluid which is equal to $K_{f}$ in active zone and is variable in inactive zone of the film. In this area the thermal conductivity is given by

$$
K(\theta)=K_{a}-\frac{B(\theta)}{B}\left(K_{a}-K_{f}\right)
$$

In Eq. (30), represents the effective bearing length covered with lubricant. It can be obtained by using continuity equation.

$$
B(\theta)=\frac{H^{\star}\left(\theta_{\text {cav }}\right)}{H^{\star}(\theta)} B
$$

For the outer surface and lateral surface of bush, the free convection and radiation hypothesis gives

$$
\begin{gathered}
\frac{\partial T^{\star}}{\partial r^{\star}} \|_{r^{\star}=R_{b}^{\star}}=-N u\left(T^{\star} \|_{r^{\star}=R_{b}^{\star}}-T_{a}^{\star}\right) \\
\frac{\partial T^{\star}}{\partial z^{\star}} \|_{z^{\star}= \pm B / 2 R}=-N u\left(T^{\star} \|_{z^{\star}= \pm B / 2 R}-T_{a}^{\star}\right)
\end{gathered}
$$

where $N u=h_{b} R / K_{b}$.

\subsection{Viscosity- temperature relation}

The variation of lubricant viscosity with temperature can be represented by Walther equation and written as

$$
\log \left[\log \frac{\mu}{\rho}+0.6\right]=k_{1}-k_{2} \log (T)
$$

where $k_{1}$ and $k_{2}$ are constants.

\subsection{Load carrying capacity}

The fluid film force and its attitude angle are given as:

$$
W=\sqrt{W_{1}^{2}+W_{2}^{2}}, \phi=\arcsin \left(\frac{W_{2}}{W}\right)
$$

where $W_{1}=-\frac{2 R^{4} \mu_{0} \omega}{C^{2}} \int_{0}^{2 R} \int_{0}^{B / 2 R} E(p) \cos \theta d z d \theta, W_{2}=$ $-\frac{2 R^{4} \mu_{0} \omega}{C^{2}} \int_{0}^{2 R} \int_{0}^{B / 2 R} E(p) \sin \theta d z d \theta$.

\subsection{Friction force and friction coefficient}

The shear stress along journal surface is expressed as [11]

$$
\tau=\mu \frac{\partial u}{\partial y}\left\|_{y=0}-\eta \frac{\partial^{3} u}{\partial y^{3}}\right\|_{y=0}
$$

The friction force can be obtained by integrating shear stress around journal surface and it is written as

$$
F_{r}=\frac{2 \omega \mu_{0} R^{3}}{C} \int_{0}^{2 \pi} \int_{0}^{B / 2 R}\left[\mu\left(\frac{1}{H}+\frac{h}{2 R} \frac{\partial P}{\partial \theta}\right)\right] d z d \theta .
$$

The coefficient of friction can be obtained by dividing friction force by film force

$$
C_{f}=\frac{F_{f} R}{W C}
$$

\subsection{Side leakage flow}

The side leakage flow for a journal bearing is given as

$$
\begin{aligned}
& Q=\frac{-C R^{2} \omega}{6} \int_{0}^{2 \pi} \frac{1}{\mu} \\
& \left.\frac{\partial E(p)}{\partial z}\right|_{z=B / 2 R}\left\{H^{\star 3}-12 H^{\star} l^{2}+24 l^{3} \tanh \left(\frac{H^{\star}}{2 l}\right)\right\} d \theta .
\end{aligned}
$$

\section{Multigrid method}

The fluid velocity components and their derivatives are evaluated for the assumed initial pressure distribution. Also, energy equation and heat transfer equation are solved simultaneously, thus producing temperature field in oil and bushing. Further, the cross film average temperature can be obtained as

$$
T^{\star}{ }_{a v}\left(\theta, z^{\star}\right)=\int_{0}^{1} T_{a v}^{\star}\left(\theta, y^{\star}, z^{\star}\right) d y^{\star}
$$

The modified Reynolds equation is solved by Multigrid method then new pressure distribution is yielded. The discretized equation can be written in more general form as

$$
A^{h} u^{h}=f^{h}
$$

where $A$ is a linear operator, $u$ is the solution, $f$ is the right hand side and $h$ is the mesh size.

The algorithm for $V$ cycle multigrid method as discussed in [25-29] and is given as

- $\quad$ Relaxing $A^{h} u^{h}=f^{h}, 2$ times with initial guess $u_{0}^{h}$

- $\quad$ Compute the residual $r^{h}=f^{h}-A^{h} u^{h}$

- $\quad$ Restrict $f^{2 h}=I^{2 h} r^{h}$, to zeros to get $u^{2 h}$

- $\quad$ Relaxing $A^{2 h} u^{2 h}=f^{2 h}, 2$ times with guess $u^{2 h}$

- $\quad$ Compute the residual, $r^{2 h}=f^{2 h}-A^{2 h} u^{2 h}$

- $\quad$ Restrict, $f^{4 h}=I_{2 h}^{4 h} r^{2 h}$, to zeros to get $u^{4 h}$

$$
\text { - }
$$

- $\quad$ Solve the error equation, $e^{H}=\left(A^{H}\right)^{-1} f^{H}$ 
(1)

- $\quad$ Interpolate $e^{2 h} \leftarrow I_{4 h}^{2 h} u^{4 h}$.

- $\quad$ Correcting the fine grid approximation $u^{2 h} \leftarrow u^{2 h}+$ $e^{2 h}$

- Interpolate $e^{h} \leftarrow I_{2 h}^{h} u^{2 h}$.

- $\quad$ Correcting $u^{h} \leftarrow u^{h}+e^{h}$.

The initial approximations are taken on finest grid and two iterations of Gauss-Seidel method is applied on finest grid for smoothing errors. To transfer the calculated residual to next coarse grid level, the half weighting restriction is used. Repeating the procedure till coarsest grid level reaches to single point. The solution is obtained at coarsest level. The bilinear interpolation is applied to prolongation of solution obtained from coarsest level to the next fine grid level and then applying two time Gauss- Seidel iterations. Repeating the procedure until original finest grid level is reached. This is referred to as one V-cycle. The iterations are repeated until it reaches to convergence.

\section{Flowchart}

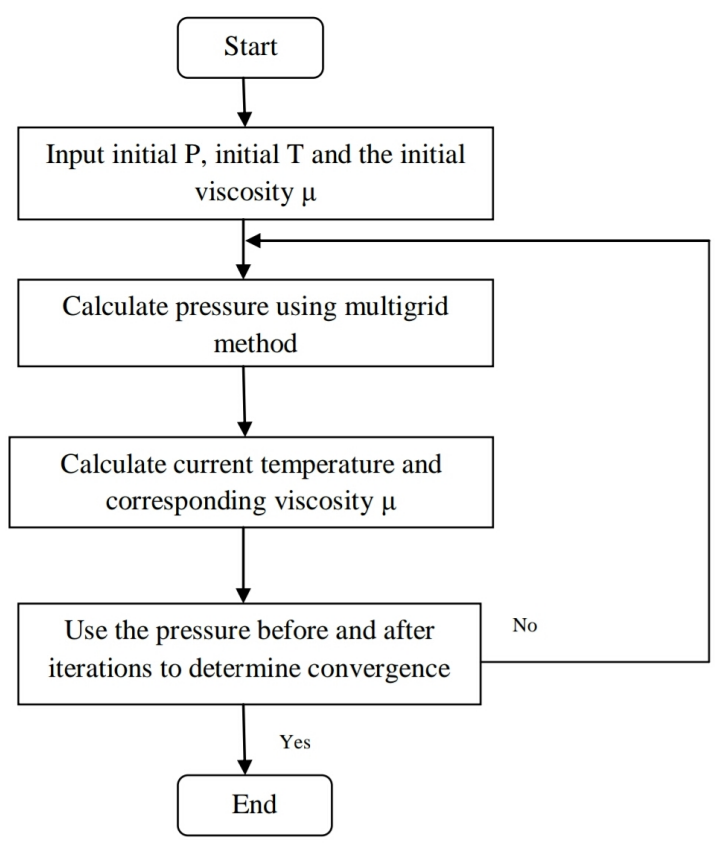

Table 1: Bearing variables used in the analysis

\begin{tabular}{ll}
\hline Bearing length, $\mathrm{B}(\mathrm{m})$ & 0.028 \\
Radial clearance, $\mathrm{C}(\mathrm{m})$ & $5.2 \times 10^{-5}$ \\
Bush specific heat, $\mathrm{c}_{b}\left(\mathrm{~J} \mathrm{~kg}{ }^{-1} \mathrm{~K}^{-1}\right)$ & 465 \\
Lubricant specific heat, $\mathrm{c}_{f}\left(\mathrm{~J} \mathrm{Kg}^{-1} \mathrm{~K}^{-1}\right)$ & 2022 \\
Shaft radius, $\mathrm{R}(\mathrm{m})$ & 0.04 \\
Bush outside diameter, $\mathrm{R}_{b}(\mathrm{~m})$ & 0.043 \\
Heat transfer coefficient, $\mathrm{h}_{b}\left(\mathrm{~W} \mathrm{~m}^{-2} \mathrm{~K}^{-1}\right)$ & 230 \\
Air thermal conductivity, $\mathrm{K}_{a}\left(\mathrm{~W} \mathrm{~m}^{-1} \mathrm{~K}^{-1}\right)$ & 0.025 \\
Bush thermal conductivity, $\mathrm{K}_{b}\left(\mathrm{~W} \mathrm{~m}^{-1} \mathrm{~K}^{-1}\right)$ & 49 \\
Lubricant thermal conductivity, $\mathrm{K}_{f}\left(\mathrm{~W} \mathrm{~m}^{-1} \mathrm{~K}^{-1}\right)$ & 0.141 \\
Rotational speed, $\mathrm{n}(\mathrm{rpm})$ & 3300 \\
Lubricant inlet pressure, $\mathrm{P}_{0}(\mathrm{~Pa})$ & $2 \times 10^{4}$ \\
Lubricant inlet temperature, $\mathrm{T}_{0}(\mathrm{~K})$ & 333 \\
Bush density, $\rho_{b}\left(\mathrm{~kg} \mathrm{~m}^{-3}\right)$ & 7800 \\
Inlet attitude angle, $\left(^{\circ}\right)$ & 24 \\
$\left.k_{1}\right)$ & 7.8212 \\
$k_{2}$ & 3.0145 \\
\hline
\end{tabular}

\section{Results and discussion}

The effect of surface roughness for thermo-hydrodynamic analysis of journal bearings extended to couple stress lubricants with high polymer additives is presented. The modified Reynolds and energy equations are solved by finite difference method i.e. multigrid method.

Couple stress mainly depends on the polar additives in the lubricant. The presence of micro structure in the fluid film influences the couple stress of the fluid which in turn increases the fluid pressure. The increase in film pressure results in an increase in the load carrying capacity. It is observed that film pressure is high for a low eccentricity ratio. This shows that load carrying capacity is high for a low eccentricity ratio. Minimum fluid film thickness increases for the bearing operate under couple stress lubricants. Stiffness and damping coefficients are larger than the couple stress fluids lubricated bearings and threshold speed improves when the bearings are lubricated by couple stress lubricants.

The temperature contour in the centre plane at the eccentricity ratio 0.3 and roughness parameter 0.1 is shown in Fig. 2. The journal bearing variables used in computer simulations to the flow with eccentricity ratio for different couple stress parameters are listed in Table 1. The shaft temperature and the maximum oil temperature are affected due to couple stress in fluids for different eccentricity ratios are shown in Figs. 3 and 4. It is observed that maximum oil temperature and shaft temperature decreases on increasing $l^{\star}$, and it increases with the addition of roughness parameter 0.1 , the influence of couple stress is more significant at a higher eccentricity ratio is 


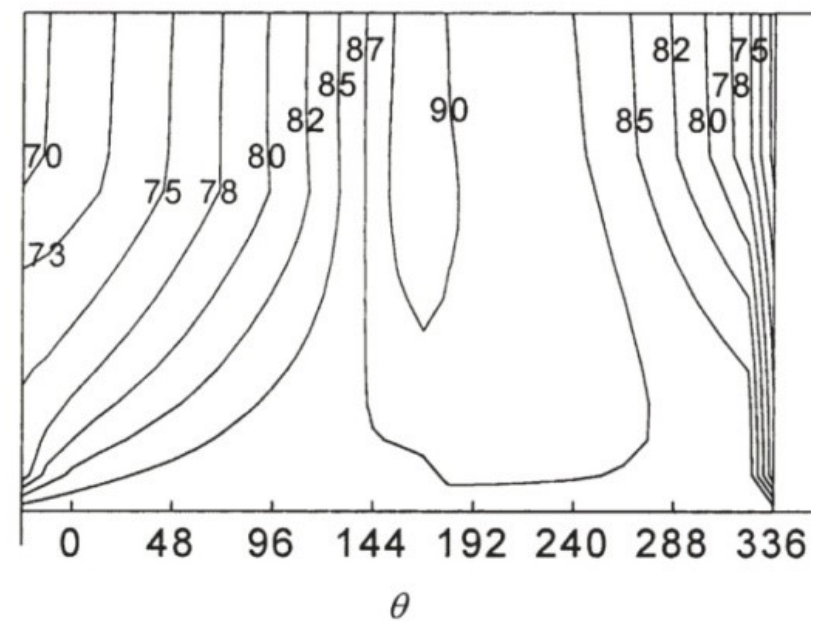

Fig. 2: Temperature contour in the centre plane at eccentricity ratio 0.3 and roughness parameter 0.1

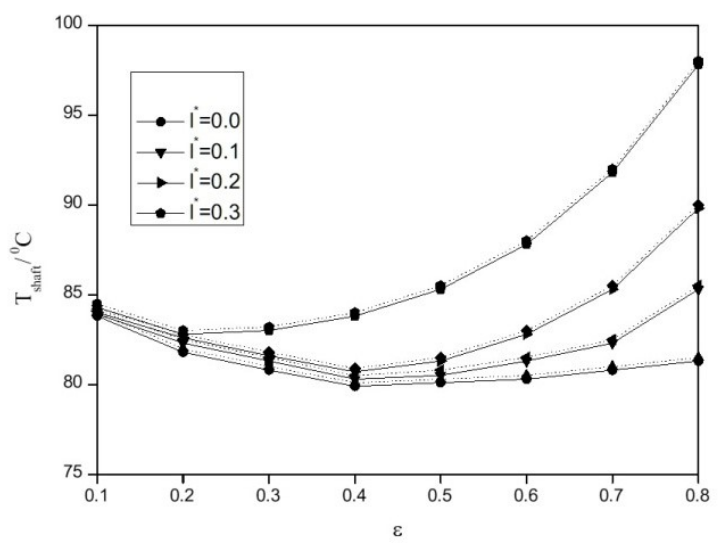

Fig. 3: Shaft temperature versus eccentricity ratio for different values of the couple stress parameter with roughness parameter 0.1

predicted. Fig. 5 shows that for any eccentricity ratio, load capacity increases with increasing $l^{\star}$ and roughness parameter 0.1 load capacity increases for all values of $l^{\star}$.

The friction force remains in increasing trend with increasing $l^{\star}$ and it increases with roughness parameter 0.1 is depicted in Fig. 6, while Fig. 7 shows that friction coefficient decreases with increase in $l^{\star}$ but it is seen that friction coefficient increases for the roughness parameter 0.1. The variation in the side leakage flow with eccentricity ratio for different couple is showed in Fig. 8. It shows that the side leakage flow of couple stress with roughness parameter 0.1 is almost same as that for different couple stress parameter $l^{\star}$.

To verify the accuracy of the present model, the circumferential pressure distribution of the lubricant film with smooth case is compared with that of surface rough-

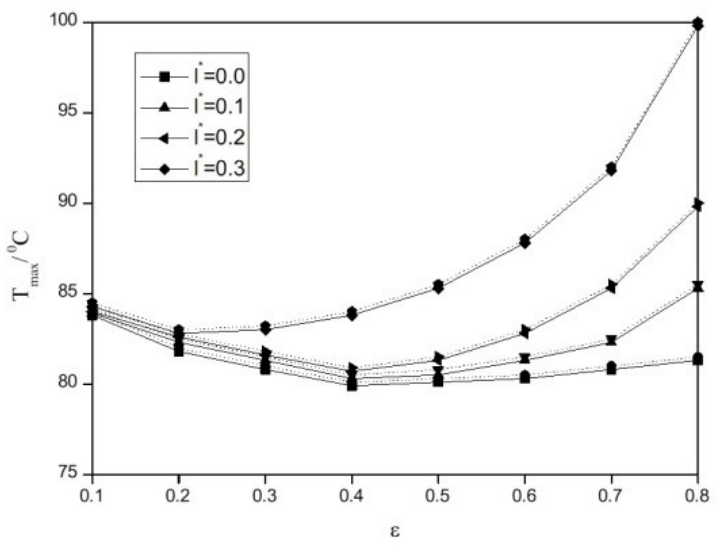

Fig. 4: Maximum temperature versus eccentricity ratio for different values of the couple stress parameter with roughness parameter 0.1

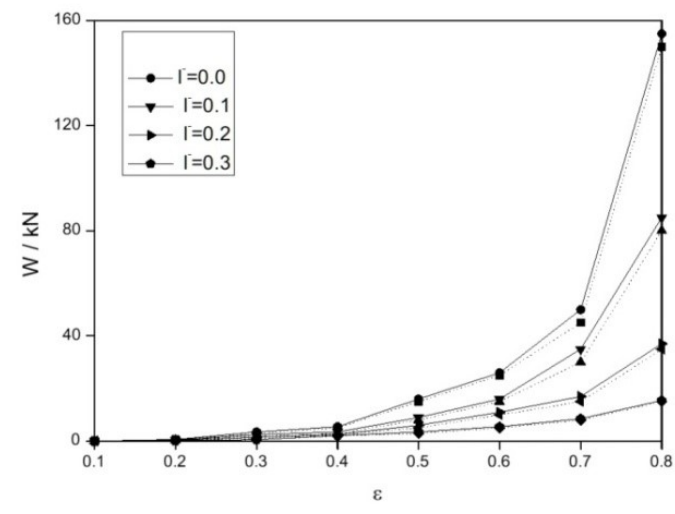

Fig. 5: Load capacity versus eccentricity ratio for different values of the couple stress parameter with roughness parameter 0.1

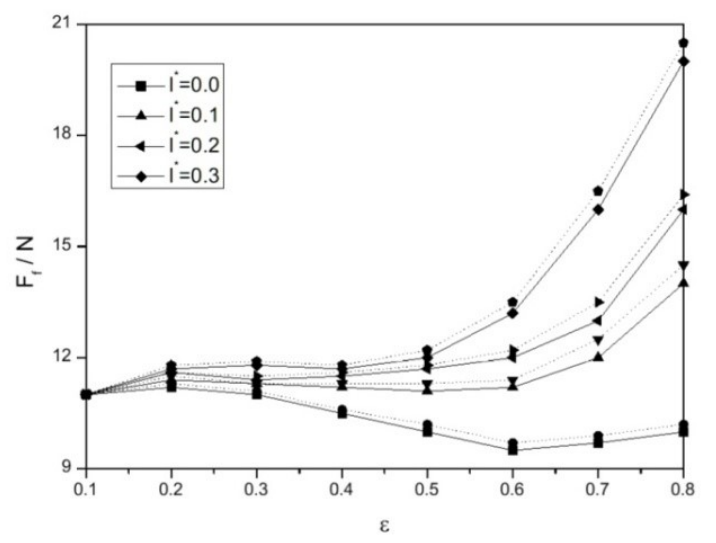

Fig. 6: Friction force versus eccentricity ratio for different values of the couple stress parameter with roughness parameter 0.1 


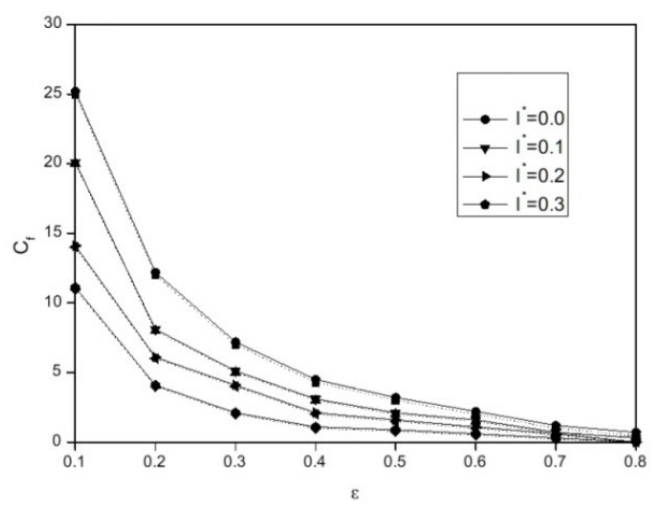

Fig. 7: Friction coefficient versus eccentricity ratio for different values of the couple stress parameter with roughness parameter 0.1

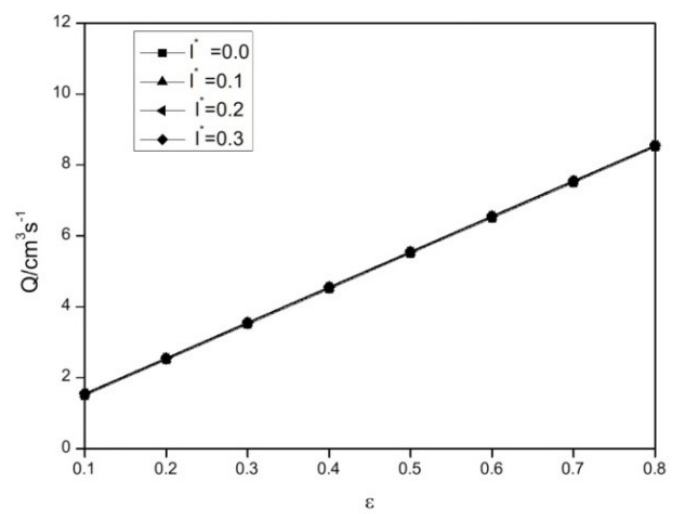

Fig. 8: Side leakage versus eccentricity ratio for different values of the couple stress parameter with roughness parameter 0.1

ness. Multigrid method is used to minimize the errors and find the solution in fast and efficient way. It is observed that, the shape of temperature contour for is same as for $l^{\star}=0.3$ but in case of temperature field for $l^{\star}=0.3$ it is lower than that for $l^{\star}=0.0$. This shows that couple stress fluids yields less heat dissipation than that of Newtonian fluids.

\section{Conclusion}

The following conclusions are made from this study.

1. The bearing temperature field is affected due to the application of polymeric additives and the presence of surface roughness. The maximum oil temperature and the shaft temperature become lower at higher eccentricity ratios.
2. The couple stress effects produce a higher load capacity, higher friction force and a lower friction coefficient but the presence of surface roughness enhances the coefficient of friction. The side leakage flow almost remains constant for different values of couple stress parameter and surface roughness.

3. The rate of convergence of the method used is more compared to the other finite difference methods.

4. The surface roughness effect case is comparable with the earlier smooth case.

\section{A Appendix}

\section{Velocity components and derivatives}

Introducing the dimensionless variables $y^{\star}=\frac{y}{H}, H^{\star}=\frac{H}{C}=$ $1 \epsilon \cos \theta$ and $l^{\star}=\frac{l}{C}$ in Eqs. (8) and (9), it becomes

$$
\begin{aligned}
& u^{\star}=\left(1-y^{\star}\right)+\frac{1}{2 \mu^{\star}} \frac{\partial p^{\star}}{\partial \theta}\left\{H^{\star 2} y^{\star}\left(y^{\star}-1\right)\right. \\
& \left.+2 l^{\star}\left[1-\left(\cosh \frac{2 H^{\star} y^{\star}-H^{\star}}{2 l^{\star}}\right)\left(\cosh \frac{H^{\star}}{2 l^{\star}}\right)^{-1}\right]\right\} \\
& \frac{\partial u^{\star}}{\partial y^{\star}}=-1+\frac{1}{2 \mu^{\star}} \frac{\partial p^{\star}}{\partial \theta}\left\{H^{\star}\left(2 y^{\star}-1\right)\right. \\
& \left.-2 l^{\star} H^{\star}\left(\sinh \frac{2 H^{\star} y^{\star}-H^{\star}}{2 l^{\star}}\right)\left(\cosh \frac{H^{\star}}{2 l^{\star}}\right)^{-1}\right\},
\end{aligned}
$$

$\frac{\partial^{3} u^{\star}}{\partial y^{\star 3}}=-\frac{H^{\star} 3}{\mu^{\star} l^{\star}} \frac{\partial p^{\star}}{\partial \theta}\left(\sinh \frac{2 H^{\star} y^{\star}-H^{\star}}{2 l^{\star}}\right)\left(\cosh \frac{H^{\star}}{2 l^{\star}}\right)^{-1}$

$$
\begin{aligned}
& w^{\star}=\frac{1}{2 \mu^{\star}} \frac{\partial p^{\star}}{\partial z^{\star}}\left\{H^{\star 2} y^{\star}\left(y^{\star}-1\right)\right. \\
& \left.+2 l^{\star}\left[1-\left(\cosh \frac{2 H^{\star} y^{\star}-H^{\star}}{2 l^{\star}}\right)\left(\cosh \frac{H^{\star}}{2 l^{\star}}\right)\right]\right\}
\end{aligned}
$$

$$
\begin{gathered}
\frac{\partial w^{\star}}{\partial y^{\star}}=\frac{1}{2 \mu^{\star}} \frac{\partial p^{\star}}{\partial z^{\star}}\left\{H^{\star 2}\left(2 y^{\star}-1\right)\right. \\
\left.-2 l^{\star} H^{\star}\left(\sinh \frac{2 H^{\star} y^{\star}-h^{\star}}{2 l^{\star}}\right)\left(\cosh \frac{H^{\star}}{2 l^{\star}}\right)^{-1}\right\} \\
\frac{\partial^{3} w^{\star}}{\partial y^{\star 3}}=-\frac{H^{\star 3}}{\mu^{\star} l^{\star}} \frac{\partial p^{\star}}{\partial z^{\star}}\left(\sinh \frac{2 H^{\star} y^{\star}-H^{\star}}{2 l^{\star}}\right)\left(\cosh \frac{H^{\star}}{2 l^{\star}}\right)^{-1}
\end{gathered}
$$




\section{Nomenclature}

$B$ Bearing width (m)

$\mathrm{c}_{b}$ Bush specific heat $\left(\mathrm{J} \mathrm{kg}^{-1} \mathrm{~K}^{-1}\right)$

$\mathrm{c}_{f}$ Lubricant specific heat $\left(\mathrm{J} \mathrm{kg}^{-1} \mathrm{~K}^{-1}\right)$

C Radius clearance (m)

$\mathrm{e}_{i j k}$ Permutation tensor

$F_{f}$ Friction force (N)

$h$ Dimensional film thickness (m)

$h^{\star}$ Dimensionless film thickness, $h^{\star}=1+\epsilon \cos \theta$

$h_{b}$ Heat transfer coefficient $\left(\mathrm{Wm}^{-2} \mathrm{~K}^{-1}\right)$

$K_{a}$ Air thermal conductivity $\left(\mathrm{Wm}^{-1} \mathrm{~K}^{-1}\right)$

$K_{b}$ Bush thermal conductivity $\left(\mathrm{Wm}^{-1} \mathrm{~K}^{-1}\right)$

$K_{f}$ Lubricant thermal conductivity $\left(\mathrm{Wm}^{-1} \mathrm{~K}^{-1}\right)$

$l$ Characteristics length of the additives, (m)

$l^{\star}$ Couple stress parameter, $l^{\star}=l / C$

$M_{i j}$ Couple stress tensor in fluid

$n$ Rotational speed (rpm)

$p$ Hydrodynamic pressure $(\mathrm{Pa})$

$p^{\star}$ Dimensionless hydrodynamic pressure, $p^{\star}=p C^{2} / R^{2}$

$p_{0}$ Lubricant inlet pressure $(\mathrm{Pa})$

$Q$ Side leakage flow $\left(\mathrm{m} 3 \mathrm{~s}^{-1}\right)$

$R$ Journal radius (m)

$R_{b}^{\star}$ Dimensionless bush outside radius, $R^{\star}{ }_{b}=R_{b} / R$

$T$ Temperature (K)

$T^{\star}$ Dimensionless temperature, $T^{\star}=T / T_{0}$

$T_{0}$ Lubricant inlet temperature (K)

$T^{\star}{ }_{a}$ Dimensionless ambient temperature, $T^{\star}{ }_{a}=T_{a} / T_{0}$

$T^{\star} \max$ Dimensionless maximum bearing temperature,

$T^{\star} \max =T \max / T 0$

$T_{\text {mix }}^{\star}$ Dimensionless mixing temperature of recirculating

fluid and supply oil, $T^{\star}$ mix $=T \operatorname{mix} / T 0$

$U$ Shaft linear speed $\left(\mathrm{m} \mathrm{s}^{-1}\right)$

$u^{\star}, v^{\star}, w^{\star}$ Dimensionless components of the lubricant velocity in the $x, y$ and $z$ directions, respectively,

$\mathrm{u}^{\star}=u / U, v^{\star}=v / U, w^{\star}=w / U$

$W 1, W 2$ Fluid film forces in the $x$ and $z$ directions (N)

$x, y, z$ Coordinates $(\mathrm{m})$

$\epsilon$ Eccentricity ratio

Inlet attitude angle

$\theta, y^{\star}, z^{\star}$ Dimensionless coordinates, $\mathrm{q}=x / R, y^{\star}=y / h, z^{\star}=z / R$

$\theta, z^{\star}, r$ Dimensionless coordinates, $\mathrm{q}=\chi / R, z^{\star}=z / R, r^{\star}=r / R$

$\theta_{c a v}$ Location of the starting of cavitation zone

$\eta, \eta^{\prime}$ Material constant responsible for the couple stress property ( $\mathrm{N} \mathrm{s}$ )

$\mu$ Lubricant viscosity (Pa s)

$\mu_{0}$ Inlet lubricant viscosity (Pa s)

$\mu^{\star}$ Dimensionless lubricant viscosity $\left(\mathrm{m}^{\star}=\mathrm{m} / \mathrm{m} 0\right)$

$\rho$ Lubricant density $\left(\mathrm{kg} \mathrm{m}^{3}\right)$

$\rho_{b}$ Bush density $\left(\mathrm{kg} \mathrm{m}^{3}\right)$

$\rho_{0}$ Inlet lubricant density $\left(\mathrm{kg} \mathrm{m}^{3}\right)$ $\rho^{\star}$ Dimensionless lubricant density, $\mathrm{r}^{\star}=\mathrm{r} / \mathrm{r} 0$

$\tau$ Viscous shear stress $(\mathrm{Pa})$

$\sigma_{i j}$ Stress tensor in fluid

$\omega$ Rotation vector $\left(\mathrm{rad} \mathrm{s}^{-1}\right)$

\section{References}

[1] Ferron J, Boncompain R. A study of the thermohydrodynamic performance of a plain journal bearing comparison between theory and experiments. ASME J Lubr Tech 1983; 105:422-8.

[2] Dawson D. A generalised Reynolds equation for fluid film lubrication. Int J mech Sci 1962; 4:159-70.

[3] Khonsari MM, Beaman JJ. Thermohydrodynamic analysis of laminar incompressible journal bearings. ASLE Trans 1987; 29:141-50.

[4] Ott HH, Paradissiadis G. Thermohydrodynamic analysis of journal bearings considering cavitation and reverse flow. ASME J Trib 1988; 110:439-47.

[5] Paranjpe RS, Han T. A study of the thermohydrodynamic analysis of steadily loaded journal bearings. STLE Trib Trans 1994; 37:679-90.

[6] Khonsari MM, Jang JY, Fillon M. On the generalization of thermohydrodynamic analyses for journal bearings. ASME J Trib 1996; 118:571-9.

[7] Ramesh J, Majumdar BC, Rao NS. Thermohydrodynamic analysis of submerged oil journal bearings considering surface roughness effects. ASME J Trib 1997; 119:100-6.

[8] Shi F, Wang Q. A mixed- TEHD model for journal bearing conformal contacts - Part I: model formulation and approximation of heat transfer considering asperity contact. ASME J Trib 1998; 120: 198-205.

[9] Ariman TT, Sylvester ND. Microcontinuum fluid mechanics - a review. Int J Eng Sci 1973; 11:905-30.

[10] Stokes VK. Couple stresses in fluids. Phys Fluids 1966; 9:170915.

[11] Mokhiamer UM, Crossby WA, El-Gamal HA. A study of a journal bearing lubricated by fluids with couple stress considering the elasticity of the liner. Wear 1999; 224:194-201.

[12] Lin J. Squeeze film characteristics of finite journal bearings: couple stress fluid model. Trib. Int. 1998; 31:201-207.

[13] Das NC. A study of optimum load-bearing capacity for slider bearings lubricated with couple stress fluids in magnetic fluid. Trib. Int. 1998; 31:393-400.

[14] Bujurke NM, Kudenatti RB, Awati VB. Effect of surface roughness on squeeze film poroelastic bearings with special reference to synovial joints. Mathematical Biosciences 2007; 209:76-89.

[15] Naduvinamani NB, Hiremath PS, Gurubasavaraj G. Surface roughness effects in a short porous journal bearing with a couple stress fluids. Fluid Dynamics research 2002; 31:333354.

[16] Xiao LW, Zhu KQ, Wen SZ. Thermohydrodynamic analysis of journal bearings lubricated with couple stress fluids. Tribology international 2001; 34: 335-343.

[17] Christensen H. Stochastic model for hydrodynamic lubrication of rough surfaces. Proceedings of the institution of mechanical engineers 1969; 184:1013-26. 
[18] Tucker PG, Keogh PS. A generalized computational fluid dynamics approach for journal bearing performance prediction. Inst Mech Eng Proc Part 3 1994; 209:99-108.

[19] Yang P, Rodkiewica CM. On the numerical analysis to the thermal elastohydrodynamic lubrication of a tilting pad inclusive of side leakage. STLE Trib Trans 1992; 40:259-66.

[20] Dowson D, Hudson J, Hunter B, March C. An experimental investigation of the thermal equilibrium of steadily loaded journal bearings. Proc Inst Mech Eng 1967; 101(3B):70-80.

[21] Spikes HA. The behaviour of lubricants in contacts: current understanding and future possibilities. J Proc Inst Mech Eng 1994; 28:3-15.

[22] Oliver DR. Load enhancement effects due to polymer thickening in a short model journal bearing, J Non-Newtonian Fluid Mech 1988; 30:185-96.

[23] Wang XL, Zhu KQ, Wen SZ. Thermohydrodynamic analysis of journal bearings lubricated with couple stress fluids. Tribology international 2001; 34:335-343.
[24] Awati VB, Kengangutti A, Mahesh Kumar NM. Multigrid method for the solution of combined effect of viscosity variation and surface roughness on the squeeze film lubrication of journal bearings. Journal of Mechanical Engineering 2017; 46:1-8.

[25] Kudenatti RB, Basti DP, Bujurke NM. Numerical solution of the MHD Reynolds equation for squeeze-film lubrication between two parallel surfaces. Applied Mathematics and computation. 2012; 218: 9372-9382.

[26] Kudenatti RB. Multigrid solution of modified Reynolds equation incorporating poroelasticity and couple stresses. Journal of Porous Media 2007; 10: 125-136.

[27] Hackbush, W. and Trottenberg, U. (Eds.), Multigrid Methods, Lecture Notes in Mathematics, 960, Springer, Berlin, 1986.

[28] Wesseling, P., Introduction to Multigrid Methods, John Wiley \& sons, 1992

[29] William L. Briggs, Van Emden Hensen and Steve F. McCormick, A Multigrid tutorial, $2^{\text {nd }} \mathrm{Ed}, 2000$. 